Voix et Images

voixetimages

\title{
L'émergence des écrits des femmes dans les Écrits du Canada français 1954-1973
}

\section{Marie-José des Rivières}

Volume 15, numéro 2 (44), hiver 1990

Pratiques illicites

URI : https://id.erudit.org/iderudit/200840ar

DOI : https://doi.org/10.7202/200840ar

Aller au sommaire du numéro

Éditeur(s)

Université du Québec à Montréal

ISSN

0318-9201 (imprimé)

1705-933X (numérique)

Découvrir la revue

Citer cet article

des Rivières, M.-J. (1990). L'émergence des écrits des femmes dans les Écrits du Canada français 1954-1973. Voix et Images, 15(2), 269-276.

https://doi.org/10.7202/200840ar d'utilisation que vous pouvez consulter en ligne.

https://apropos.erudit.org/fr/usagers/politique-dutilisation/ 


\section{L'émergence des écrits des femmes dans les Écrits du Canada français 1954-1973}

\section{par Marie-José des Rivières, Musée de la civilisation}

En 1954 naissent les Écrits du Canada français, une nouvelle instance de production et de consécration de la littérature québécoise. Cette entreprise commune d'un groupe d'[auteurs, de journalistes et de critiques] est un périodique qui veut servir la littérature française en Amérique [...] permettre [...] le dégagement des tendances et des formes les plus actuelles [de l'imaginaire] [...] et aborder l'étude des grands courants de pensée ${ }^{1}$.

Tentant de re-situer les écrits des femmes dans l'histoire littéraire, nous pouvons nous demander quelle place cette collection d'œuvres libres ${ }^{2}$ fait aux productions des femmes et dans quels genres se sont surtout manifestées les écrivaines durant les vingt premières années de publication du collectif (1954-1973). Une observation du contenu des textes sera indicative de leur spécificité, de leur évolution et des effets de lecture produits par limplication des femmes dans ce périodique.

\section{Une recherche à perspective féministe}

La critique féministe dénonce les tabous mentaux, les répressions psychologiques et les limites apportées à la créativité et à la liberté de pensée ou d'expression dont les femmes ont le plus souvent été les victimes. Dans son article-synthèse sur les théories féministes, Jeannette Laillou Savona ajoute que des écrits qui avaient [...] été considérés comme inférieurs selon les canons officiels sont soudains apparus profondément riches et signifiants à celles qui en découvraient les différences thématiques et esthétiques et qui se mettaient à les comparer [...] aux critères de gouts dominants (masculins), de leurs époques d'origine ${ }^{3}$.

L'hypothèse d'une tradition propre aux femmes peut être rapprochée du postulat inspiré de Virginia Wolf d'une poétique spécifiquement féminine dans les œuvres signées par des femmes. Cette altérité serait reliée à une spécificité tout au moins sociale et culturelle du destin des femmes, qui ont longtemps été écartées de l'éducation, du pouvoir et de la propriété.

Il nous apparaît intêressant de détecter, un peu à la manière de Béatrice Didier dans son essai l'Écriture femme, ce qui démarque les [textes des]

1 Paul Beaulieu, Robert Charbonneau, Roger Duhamel et al., « Présentation „, Ecrits du Canada français [dorénavant E.C.F.], vol. I, no $, 1,1954$, p. 17.

2 Ibid.

3 Jeannette Laillou Savona, « Le féminisme et les études littéraires en France et en Amérique du Nord *, Littérature, n89, février 1988, p. 117-118. 
écrivaines de [ceux de] leurs contemporains, posant l'hypothèse d'une différence qui se dessine d'une auteure à l'autre, [distinction qui] finit par créer un réseau intertextuel spécifique 4 .

\section{La présence des femmes dans ce périodique}

Disons tout d'abord qu'il n'est pas surprenant que les textes des femmes aient mis du temps à occuper une place soutenue dans les Écrits. Jeanne Lapointe et Anne Hébert étaient les deux seules femmes parmi les 28 personnes qui ont fondé la revue, regroupement dont le noyau était essentiellement le groupe de Cité libre. Pas d'écrivaines dans le premier comité de rédaction formé de Robert Élie, Jean-Louis Gagnon, Gilles Marcotte, Gérard Pelletier, Paul Toupin et Pierre Elliott Trudeau. La première collaboration féminine au comité de rédaction aura lieu en 1971, avec la présence de Gertrude LeMoyne, qui demeurera l'unique femme du groupe; elle le quittera en 1983. Dans les 12 premiers numéros, constitués surtout de nouvelles et de récits, d'essais et de pièces de thêâtre, les productions de femmes - 10 sur 55 - couvrent à peine $19 \%$ des œuvres, ceci jusqu'en 1962. Les premières contributions des écrivaines sont des poèmes (de Hélène J. Gagnon, de Marie-Claire Blais, de Suzanne Paradis), ainsi que du théâtre (d'Anne Hébert); pas un essai, à l'exception, peut-être, d'un récit de voyage en Chine de la journaliste Hélène Gagnon, en 1961. Dans la littérature québécoise en général, les femmes écrivaient peu d'essais. C'est seulement sept ans après les débuts du périodique qu'on peut lire la première nouvelle signée par une femme, texte intitulé "Ma sœur" d'Andrée Thibault; à partir de 1961, les textes des femmes emprunteront très souvent ce genre ${ }^{5}$.

Cela dit, les Écrits, avec leurs six nouvelles par année, en moyenne, ne sont pas le principal lieu de diffusion de la nouvelle littéraire dans les années soixante. La palme revient à un magazine féminin à grand tirage, la revue Châtelaine, qui publiait chaque mois deux nouvelles de douze à seize pages dactylographiées. Déjà, la nouvelle et le conte n'entraient pas dans les principaux courants de l'édition. En 1964, raconte Jacques Hébert, ancien directeur des Éditions du Jour, les contes se vendent mal, plus mal encore que les romans. Et les contes d'un jeune auteur inconnu, c'est la catastrophe ${ }^{6}$. Au même moment, Châtelaine fait de la publication des nouvelles et des contes une véritable spécialité. Cependant, nous ont confié Fernande Saint-Martin, rédactrice en chef du magazine jusqu'en 1973, et Gertrude LeMoyne, lectrice consultante ${ }^{7}$, Châtelaine n'acceptait pas toutes les nouvelles qu'on lui faisait parvenir. Et même pas toutes les excellentes nouvelles. Il y avait deux catégories de textes de qualité qu'elle rejetait - en recommandant toutefois à leurs auteurs et auteures de les faire parvenir... aux Écrits du Canada français. Ce qui était inadmissible à

4 Béatrice Didier, l'Écriture femme, Paris, P.U.F., 1981, p. 119.

5 On trouvera en bibliographie les titres des nouvelles signées par des auteures dans les Écrits du Canada francais entre 1954 et 1973; notre étude porte sur l'ensemble de ces nouvelles.

6 Jacques Hébert, «Qui est Roch Carrier? Son éditeur répond *, PEnvers du décor, vol. III, no 1 , octobre 1970 , p. 3.

7 Fernande Saint-Martin [interviewée par Marie-José des Rivières], automne 1979; Gertrude LeMoyne [interviewée par Marie-José des Rivières], hiver 1980. 
l'homogénéité de Châtelaine, c'était quelques textes trop "savants", c'est-à-dire difficiles d'accès pour un public de masse mais surtout les textes trop "noirs". C'est cette deuxième catégorie qui nous intéresse aujourd'hui. Nous avons devant les yeux un processus d'exclusion, issu d'une certaine censure, ou tout au moins de normes, de choix précis. Nous ne disons pas que tous les textes des Écrits ont d'abord été soumis à Châtelaine; cependant, cette information sur le rejet des textes sombres (qui a d'ailleurs été confirmée par Claude Jasmin dans son autobiographie ${ }^{8}$ ) mérite d'être interprétée, et la recommandation de publication dans les Ecrits fournit des indices susceptibles de nourrir notre recherche.

Nous voulons faire une lecture féministe de ces nouvelles écrites par des femmes, c'est-à-dire relire les textes avec un regard attentif qui comble les oublis, rend aux femmes leur part d'histoire, et prépare à une poétique des écrits de femmes. Cette approche considère en particulier les rapports des personnages féminins à la société qui les entoure et les relations entre les sexes, qu'elle observe d'un point de vue critique et politique.

Il faut tout d'abord dire que les vingt-sept nouvelles écrites par des femmes durant ces vingt premières années des Ecrits du Canada français (1954-1973) mettent presque toujours en scène des héroïnes, mais cette caractéristique peut aussi se rattacher à l'évolution du roman québécois contemporain écrit par des femmes, en particulier le roman autobiographique signé par des écrivaines des années soixante-dix, dans lequel se développe une thématique axée sur les confidences et la vie privée.

\section{Le destin, sombre, des héroïnes}

Ce destin des héroïnes est d'abord illustré, dans les Écrits, par le récit d'une religieuse malheureuse qui s'est trompée de vocation et qui effectue, lors d'une démarche de prise de conscience, un pèlerinage dans son enfance. La pensée, scandaleuse, de sortir du couvent, la plonge dans le désarroi. Ai-je mesuré l'ampleur de mon geste? [...] moi qui ai été élevée, éduquée dans le souci de l'opinion de mon entourage, comment en suis-je là maintenant ? $^{9}$

Le second texte est lui aussi axé sur les souvenirs et la soumission. La "Chronique d'une enfance", de Françoise Cholette-Pérusse, raconte l'ennui d'une petite fille de neuf ans à qui l'on interdit les jeux d'aventures auxquels s'adonnent les garçons. À défaut de pouvoir courir les bois avec son ami Sylvain, Anne-Marie rêve de lui et confie ce premier amour à son journal intime... calepin qu'une cousine indiscrète lit devant tout le monde réuni à une fête, le dernier jour de vacances. Insulté, le garçon la gifle; et c'est avec des larmes de rage qu'elle s'enfuit - scène finale - au milieu des quolibets. Elle emprunte un chemin dangereux sur le bord du lac où elle voudrait se perdre ${ }^{10}$.

Ces récits, en apparence anodins, projettent presque tous une immense sensation d'impuissance. Déjà, les titres sont évocateurs: "Trop tard", "Les

8 Claude Jasmin, Jasmin, Montréal, Claude Langevin éditeur, 1970, p. 106.

9 Andree Thibault, «Ma sœur «, E.C.F., $n^{\circ} 10,1961$, p. 371.

10 Françoise Cholette-Pérusse, «Chronique d'une enfance », E.C.F., n` 12, 1962, p. $253-275$. 
incompris", "Les solitudes humaines" d'Alice Poznanska; "Les souvenirs usés" de Christiane Bacave; "La laide" de Micheline Gagnon; "L'ombre" de Carol Dunlop Hébert; "Récit d'une errance" de Thérèse Renaud. Ils se terminent presque tous sur des images de mort: l'héroïne se suicide, en rêve ou en réalité, des femmes se font des misères et se tuent ou encore elles assassinent leurs maris. Mais avant d'en venir là, elles subissent d'abord le mépris et l'extrême violence des hommes qui les entourent. Par exemple, le récit de Léa Pétrin, "Violence chez Sam Careau", raconte l'histoire d'une jeune fille violée qui, après quatre longs mois de solitude et de silence, apprend la nouvelle de sa grossesse à son père. Blessé dans son orgueil, il ne trouve rien de mieux à faire que de la battre. Il scande chaque coup d'une injure. Il est d'autant plus déchaîné que l'homme qui s'est jeté sur sa fille l'a déjà insulté, lui; une fille sur qui un échevelé, un repris de justice s'est soulagé, pense-t-il, mérite d'être battue et ensuite cachée dans le caveau à légumes, à la cave.

Anne, la mère ramasse une mèche rousse à laquelle [tiennent] des parcelles de peau et de sang et la garde dans sa main, comme si elle pouvait ainsi partager la douleur, le cauchemar de sa fille [...]. Entre deux indignités elle ne sait dire laquelle l'afflige le plus, le spectacle obscène de l'homme sans cceur ni sensibilité à qui elle est liée ou le traitement brutal infligé aux êtres autour d'elle [...] Il y a dans cette vie, pense. $t$-elle, quelque chose d'injuste. Qui donc donne à celui-là le droit de frapper et à cet autre le lot d'encaisser? Qui dispense l'autorité à l'un et l'obligation à l'autre? 11

Au matin elle conçoit un plan pour sauver sa fille mais il est trop tard. Rourque est morte: Tu diras qu'elle a culbuté dans l'escalier ${ }^{12}$, lui commande son mari qui réussit à cacher à tous la véritable cause de la mort de la jeune fille... Trois jours plus tard, soulagé, il cloue le cercueil. Mais Anna voit le bras de Sam se lever et s'abattre, se lever de nouveau et s'abattre et en profil, dans la première clarté du jour, elle aperçoit non pas le couvercle de la tombe mais le corps de Rourque [...] les bras, [les épaules], le visage et les coups semblent exalter les prieurs agenouillés, dont la voix monte ${ }^{13}$. Anna court dans la cuisine, prendre le fusil de chasse. Elle décharge l'arme comme si elle abattait un chien saisi de rage.

\section{Insatisfaction et révolte}

Les personnages féminins portent sur l'univers masculin un jugement très sévère, exprimant l'insatisfaction et la révolte. Au mieux, le masculin représente les institutions (''avocat, le marchand qui réussit, le garde-chasse, etc.). S'ils ne sont pas toujours absents et égoïstes, les maris sont orgueilleux et mesquins.

Un de ces maris possessifs décide de poser les fenêtres doubles; à l'automne on enferme les femmes dans un univers ouaté et assourdi, c'est du moins ainsi que l'héroïne de "La femme-prétexte», de Marianne Favreau, perçoit la vie.

11 Léa Pétrin, "Violence chez Sam Carreau », E.C.F., n 20, 1965, p. 163.

12 Ibid., p. 167.

13 Ibid., p. 174. 
Il se croyait fort, et habile, et invulnérable. Et moi, son public, je devais sourire, approuver, encourager. Quand il m'a demandé un chiffon propre (pour laver les vitres) je me suis levée, sachant déjà ce qui allait arriver [...]. Et le geste s'est fait presque malgré moi. J'ai présenté le chiffon d'une main, et de l'autre, j'ai poussé son pied. Ouf? la fenêtre était libérée [...]. Partie, cette ombre sur ma vie. Sans un cri. "Avec sa cervelle d'oiseau, il aurait dû voler.» Voilà tout ce que j'ai pensé. ${ }^{14}$

Mais le hasard fait que le mari ne meurt pas. Bien sûr qu'il se souvient de la main sur sa cheville... Elle pense elle-même à se suicider, mais à quoi bon si c'est pour atterrir dans les lilas étêtés du jardin. De toute façon, elle sait qu'elle mourra, car elle a un cancer. Après le procès, où le mari se montre à la fois magnanime et sordide, elle recommande à sa fille de ne jamais miser sur l'avenir, parce que la vie d'une femme reste, à ce moment, un brouillon, un croquis, une ébauche, un prétexte à sa propre inertie, à son absence.

Les personnages féminins ne peuvent s'échapper de ce monde aliénant. Rejetant la morale traditionnelle, les notions apprises dans la famille ou au pensionnat, interrogeant la rigidité des classes sociales et les dictats de leurs compagnons, les femmes de ces récits vivent un perpétuel conflit de valeurs, dont elles souffrent. Mésadaptées, révoltées, désabusées, voulant se venger des méfaits qu'on leur a fait subir, elles n'ont plus rien à perdre. Le bonheur n'existe pas dans ces récits. Et la relation amoureuse est presque impossible. Si elle est sur le point d'exister, elle est aussitôt niée par le suicide de la jeune fille, comme dans "La cloison" de Minou Petrowski, ou mise en péril par la présence d'autres personnages, parfois étranges comme la Zanaïda de "L'affaire du plat du chat " d'Andrée Maillet.

\section{Glissement vers un fantastique qui a pour thèmes la femme, l'écriture et la mort}

Nous avons vu que ces textes frappent l'imagination, n'épargnant aucun détail morbide. Mais la narration, de type réaliste, atteint bientôt un degré de saturation. Se produit alors un glissement vers le fantastique. Léclatement du fantastique, écrit André Belleau, témoigne du fait qu'une société commence à se donner à ellemême le spectacle figuré de ce qui, sourdement, profondément, la travaille ${ }^{15}$.

Ce fantastique a pour principaux thèmes la femme, l'écriture et la mort qui s'entremêlent en une sorte de cauchemar. "La femme-prétexte" - celle qui étouffe littéralement et qui tente de tuer son mari en le jetant par la fenêtre dit, à son procès, qu'elle a un grand projet de "tapisserie»; en fait, elle n'ose prononcer le vrai mot: "écriture", "roman". Tapisserie fait plus féminin 16 , et le juge, pense-t-elle, sera moins dérouté; elle plaide donc qu'elle ne veut pas mourir, en prison, avant d'avoir terminé cette œuvre. québécois au XX` siècle, Montréal, Fides, 1987, p. 7. 
Le "Triptyque» de Louise Maheux-Forcier valorise la mort comme la suprême liberté, le seul point de vue objectif, l'autre conscience, la vraie. La mort de la narratrice lui permet de se projeter à l'intérieur de ses personnages qui, à leur tour, sont arrachés du monde pour pouvoir le réinventer. Ici, la seule union possible s'effectue dans la mort.

Ailleurs, dans "Les petits trains" de Claudette Charbonneau-Tissot, les personnages qui sortent des murs sont effrayants; le père se croit obligé d'étrangler son enfant qui est trop malade, tandis que la mère a le visage et la voix de la narratrice inquiète qui se dit qu'elle est peut-être cette femme... "L'énigme du coude", de la même auteure, est une histoire tarabiscotée, pleine de coudes, justement, où la narratrice est poursuivie par le regard d'un homme qui tente de la dominer. Après que cet homme eut arraché un sein à son amie Elsa, la narratrice le tue: elle constate alors avec soulagement que les yeux de ce personnage se vitrifient et qu'elle et lui sont maintenant quittes ${ }^{17}$.

Comme l'écrit Maurice Émond:

Le monstre, c'est l'image inavouable qui vient combler un vide inacceptable, [c'est] une hantise subitement libérée. L'inexpliqué et l'inexplicable font éclater au sein du réel apprivoisé les failles, les disjonctions, les figures par où apparaissent, dans leur éclatante nudité ou leur inquiétante imprécision, les temps et les espaces maudits, de même que leurs habitants maléfiques. [...] Le récit fantastique s'adapte [donc] aux croyances et aux idéologies pour mieux les contaminer de l'intérieur, les faire jaillir et laisser le lecteur devant l'indicible. ${ }^{18}$

\section{Réflexion politique et créativité des auteures}

L'éclatement que nous venons de voir témoigne, dans la société des récits, d'un refus de ce qui, auparavant, "allait de soi "; il fait prendre conscience du malaise des personnages et de leur désir de créer une nouvelle réalité sociale. Se trouve ainsi affirmé, dans l'hétérogène de ces textes, un lien entre la réflexion politique et la créativité intellectuelle des auteures. On peut en outre postuler une continuité entre ce fantastique, qui prend le relai d'un réalisme devenu impuissant à exprimer la thématique du désespoir, et le travail, encore plus radical, de déconstruction et de transgression dans le langage qu'apportera un peu plus tard la nouvelle écriture féminine, interrogeant les habitudes linguistiques du patriarcat.

En guise de conclusion, poursuivons maintenant la comparaison que les textes sombres nous ont amenée à poser entre Châtelaine et les Écrits; nous constatons que le délire, la folie, la transgression peuvent davantage s'exprimer dans les Écrits du Canada français, une revue littéraire spécialisée, plus audacieuse mais également beaucoup plus limitée dans sa diffusion, que dans Châtelaine, un magazine de masse soumis à des impératifs commerciaux. Notre étude

17 Claudette Charbonneau-Tissot, «L'énigme du coude n, E.C.F., n 36, 1973, p. 203.

18 Maurice Émond, op. cit., p. 7-8. 
socio-sémiotique de l'ensemble des écrits de Châtelaine ${ }^{19}$, a révélé la présence d'un modèle narratif commun qui s'intitule "l'heure des choix" et qui dans son déroulement implique, après la confrontation polémique, des issues diverses - union, désunion (euphorique ou dysphorique) ou accord avec soi-même -, finales qui sont souvent tissées de compromis. Au contraire, les nouvelles des Écrits - du moins celles qui sont signées par des femmes -, se révèlent tragiques du début à la fin, et sans accommodements.

Avec les Écrits du Canada français, nous sommes paradoxalement en face d'un hétérogène, avons-nous dit, qui se nourrit d'images de révolte, les plus souvent tirées du destin de femmes ordinaires - comme les lectrices de Châtelaine - mais qui se consomme dans l'homogénéité du cercle des intellectuelles et intellectuels.

\section{Bibliographie}

BACAVE, Christiane, «Des souvenirs usés », E.C.F., nº 24, 1968, p. 123-132.

BARBEAU, Marion, * Amour fluide», E.C.F., nº 21, 1966, p. 94-96.

BOURGET, Lise, «La spirale , E.C.F., $\mathrm{n}^{\circ} 18,1964$, p. 119-128.

CHARBONNEAU-TISSOT, Claudette, «L'énigme du coude», E.C.F., $n^{\circ} 36,1973$, p. 191 203.

CHARBONNEAU-TISSOT, Claudette, «Les petits trains «, E.C.F., $\mathrm{n}^{\circ}$ 36, 1973, p. 179-189.

CHOLETTE-PÉRUSSE, Françoise, «Chronique d'une enfance *, E.C.F., n 12, 1962, p. 253 275.

DOYON, Paule, «Un procès *, E.C.F., n 37, 1973, p. 211-218.

DUNLOP-HÉBERT, Carol, «L'ombre », E.C.F., n 32, 1971, p. 50-69.

FAVREAU, Marianne, « La femme-prétexte», E.C.F., $\mathrm{n}^{\circ}$ 25, 1969, p. $21-28$.

FECTEAU, Hélène, «La vallée sacrée», E.C.F., nº 23, 1967, p. 91-112.

GAGNON-MAHONY, Madeleine, «La laide», E.C.F., $\mathrm{n}^{\circ} 25,1969$, p. 29-38.

GAGNON-MAHONY, Madeleine, «Wilfrid-le-quêteux *, E.C.F., n 25, 1969, p. 39-56.

MAHEUX-FORCIER, Louise, *Triptyque*, E.C.F., $\mathrm{n}^{\circ}$ 20, 1965, p. 177-191.

MAILLET, Andrée, «L'affaire du plat du chat », E.C.F., n 14, 1962, p. 311-343.

McGIBBON, Marcelle, «Le manège ivre «, E.C.F., $\mathrm{n}^{\circ}$ 24, 1968, p. 104-122.

NANTEL, Françoise, «Mémorial de sa rencontre, E.C.F., n 21,1966 , p. 73-82.

PÉTRIN, Léa, * Violence chez Sam Careau *, E.C.F., nº 20, 1965, p. 143-175.

PÉTROWSKI, Minou, «La cloison *, E.C.F., n 12, 1962, p. 307-362.

POZNANSKA, Alice, «Les solitudes humaines *, E.C.F., n 12, 1962, p. 277-305 [cf. «Trop tard «, p. 279-284; «Les incompris», p. 285-291; «Nez rouge», p. 292-298; «Lorganiste», p. 299-305].

19 Marie-José des Rivières, «Châtelaine et la littérature », thèse de Ph.D., Québec, université Laval, 1988, 433 f. 
RENAUD, Thérèse, « Récit d'une errance *, E.C.F., $\mathrm{n}^{\circ}$ 34, 1972, p. 125-147.

THIBAULT, Andrée, «Ma sœur *, E.C.F., nº 10, 1961, p. 310-372.

THIBOUTOT, Thérèse, «La femme du ministre , E.C.F., $\mathrm{n}^{\circ}$ 13, 1962, p. 165-185.

ERRATUM: dans notre précédent numéro, dans l'article de François Dumont, "L'Hexagone et la nationalisation de la poésie québécoise", il aurait fallu lire, au quatrième paragraphe de la p. 101, à l'extérieur au lieu de à l'intérieur. 